\title{
Formalin-casein enhances water absorbency of calcium alginate beads and activity of encapsulated Metarhizium brunneum and Saccharomyces cerevisiae
}

\author{
Katharina M. Hermann ${ }^{1,2} \cdot$ Alexander Grünberger $^{2} \cdot$ Anant V. Patel $^{1}$ (D)
}

Received: 11 June 2021 / Accepted: 6 August 2021 / Published online: 18 August 2021

(c) The Author(s) 2021

\begin{abstract}
The control of root-feeding wireworms has become more challenging as synthetic soil insecticides have been progressively phased out due to environmental risk concerns. Innovative microbial control alternatives such as the so-called attract-and-kill strategy depend on the rapid and successful development of dried encapsulated microorganisms, which is initiated by rehydration. Casein is a functional additive that is already used in food or pharmaceutical industry due to its water binding capacity. Cross-linked forms such as formalin-casein (FC), exhibit altered network structures. To determine whether FC influences the rehydration of alginate beads in order to increase the efficacy of an attract-and-kill formulation for wireworm pest control, we incorporated either casein or FC in different alginate/starch formulations. We investigated the porous properties of alginate/ starch beads and subsequently evaluated the activities of the encapsulated entomopathogenic fungus Metarhizium brunneum and the $\mathrm{CO}_{2}$ producing yeast Saccharomyces cerevisiae. Adding caseins altered the porous structure of beads. FC decreased the bead density from $(1.0197 \pm 0.0008) \mathrm{g} / \mathrm{mL}$ to $(1.0144 \pm 0.0008) \mathrm{g} / \mathrm{mL}$ and the pore diameter by $31 \%$. In contrast to casein, FC enhanced the water absorbency of alginate/starch beads by $40 \%$. Furthermore, incorporating FC quadrupled the spore density on beads containing M. brunneum and S. cerevisiae, and simultaneous venting increased the spore density even by a factor of 18 . Moreover, FC increased the total $\mathrm{CO}_{2}$ produced by M. brunneum and S. cerevisiae by $29 \%$. Thus, our findings suggest that rehydration is enhanced by larger capillaries, resulting in an increased water absorption capacity. Our data further suggest that gas exchange is improved by FC. Therefore, our results indicate that FC enhances the fungal activity of both fungi M. brunneum and S. cerevisiae, presumably leading to an enhanced attract-and-kill efficacy for pest control.
\end{abstract}

\section{Graphic abstract}
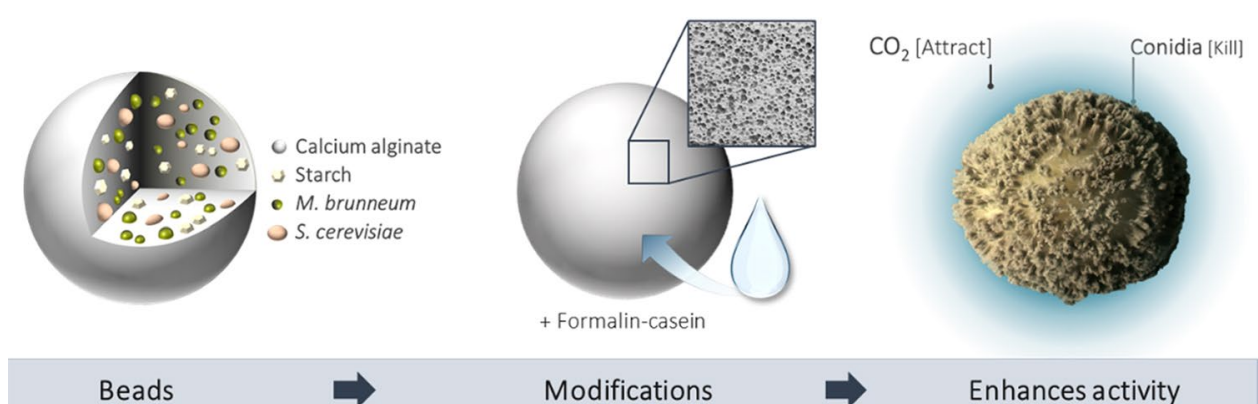

Modifications

Enhances activity

Keywords Alginate beads · Attract-and-kill · Formalin-casein · Metarhizium brunneum · Porosity · Water absorption · Saccharomyces cerevisiae

Anant V. Patel

anant.patel@fh-bielefeld.de

Extended author information available on the last page of the article 


\section{Introduction}

Wireworms (Coleoptera: Elateridae) are widespread pests that damage a variety of crops, including grain i.e. wheat, barley, maize and most vegetables such as carrot and potato. Since the mid-twentieth century wireworm damage is becoming an increasing concern in both conventional and organic potato cultivation (Poggi et al. 2018; Vernon and van Herk 2013). Wireworms can be very destructive and difficult to control. Even low populations lead to reduced quality of the harvested tubers and thus to severe economic losses (Parker and Howard 2001). Effective plant protection products are currently not available, since chemical insecticides have been restricted or abandoned. Neonicotinoids and fipronil were the main classes of insecticides routinely used and both have been restricted by the European Union (European Food Safety Authority 2013). Consequently, there is a tremendous need for control options, either conventional, or better yet, biological such as microbial pest control.

There are several approaches to microbial pest control, one of which is the attract-and-kill strategy, where pests are attracted toward an entomopathogenic pathogen (Brandl et al. 2017). Humbert et al. (2017a, b) and Pryzyklenk et al. (2017) developed an alginate bead formulation containing the $\mathrm{CO}_{2}$ producing yeast Saccharomyces cerevisiae and the entomopathogenic fungus Metarhizium brunneum (Humbert et al. 2017a, b; Przyklenk et al. 2017). Native corn starch is additionally incorporated into these beads as an indispensable filler material and nutrient supply. M. brunneum produces amylolytic enzymes and is therefore able to degrade starch, providing glucose for both fungi (Przyklenk et al. 2017). The attract-and-kill approach takes advantage of the behavior of wireworms and other soil dwelling larvae which use $\mathrm{CO}_{2}$ for locating growing roots of potential host plants (Arce et al. 2021). Dried beads are put below ground whereupon they absorb soil moisture, reactivating the encapsulated microorganisms. As a result, wireworms are attracted by the $\mathrm{CO}_{2}$ and are subsequently infected by the virulent aerial conidia of $M$. brunneum, formed on the bead surface (Brandl et al. 2017; Schumann et al. 2014). With this approach, the onset of action and the effect itself depend on the development and activity of both fungi. The development of the fungi, in turn, depends on sufficient rehydration of the dried formulation. Therefore, adequate water absorption of alginate beads and eventual water retention is crucial for a certain and reliable efficacy.

Three major approaches to modify the water absorbency of alginate gels have already been studied: I. The choice of crosslinking agents and the crosslinking time (Kim et al. 2000; Kulkarni et al. 2000), II. the enzymatic in-vitro modification of alginate (Mørch et al. 2007), and III. blending other materials such as biopolymers (Chan et al. 2008).
Biopolymers have been investigated for their capability of enhancing the water absorbency specifically of calcium alginate beads. In studies by Vreeker et al. (2008) and Fang et al. (2011) beads with gelatin, gum arabic or carboxymethylcellulose rehydrated faster. The polymers probably altered the supramolecular structure of the alginate gel during drying. More specifically, they might reduce the side-by-side aggregation of the alginate egg box junctions, resulting in less dense beads. However, as major drawback, gum arabic only reached the equilibrium swelling ratio of control alginate beads, and gelatin even decreased it. For carboxymethylcellulose, equilibrium was not even determined (Fang et al. 2011; Vreeker et al. 2008).

However, proteins generally inhere excellent functional properties and are commonly used in food and pharmaceutical industry because of their high nutritional value and emulsification, gelation, foaming and water binding capacity (Elzoghby et al. 2011; Zayas 1997). Casein, the major milk protein, is generally regarded as safe, readily available, comparatively inexpensive, non-toxic and biodegradable. Casein can be crosslinked through chemical agents such as formaldehyde, resulting in a more complex network structure and altering its mechanical properties (Elzoghby et al. 2011). The resultant formalin-casein (FC) has proven to be suitable as non-swelling tablet-disintegrant (Fekete et al. 1990; Ponchel and Duchene 1990). Nevertheless, there have been only few reports on alginate/casein hydrogel systems, which were investigated for their mechanical, release, or physico-chemical properties (Bajpai et al. 2017; He et al. 2015; Patwa et al. 2020). Patwa et al. (2020) demonstrated that amide cross-linked alginate-casein hydrogels for wound healing applications, exhibited fast swelling with high water uptake.

To the best of our knowledge no studies have been reported yet on incorporating casein or FC into calcium alginate which already contained starch as filler. Hence, the main objective of this study was to investigate how FC impacts the water absorbency of calcium alginate/starch formulations, used for attract-and-kill pest control, and whether this, in turn, enhances its effectiveness. Although starch is already included in the formulation, acting as spacer between alginate junctions, we aimed to use $\mathrm{FC}$ as rehydration additive to further enhance water absorbency.

The first part of this work elucidates the influence of casein and FC on the porous properties and the rehydration of alginate/starch beads. The second part clarifies how casein and FC, and consequently the bead properties, influence the fungal activity of M. brunneum in Kill beads or of S. cerevisiae and M. brunneum in attract-and-kill beads (A\&K beads), aiming to quickly provide a sufficient amount of attractive $\mathrm{CO}_{2}$ and entomopathogenic conidia. 


\section{Methods}

\section{Materials}

All chemical compounds used in this study were acquired from Carl Roth GmbH (Karlsruhe, Germany) and concentrations are given as $(w / w)$, unless stated otherwise.

\section{Preparation of Caseins}

Casein from bovine milk (Sigma Aldrich, USA) was grinded in a ball mill and sieved subsequently to reach a particle size $\leq 150 \mu \mathrm{m}$. Formalin-casein (FC, commercialized as EsmaSpreng, Gaerungschemie Dessau $\mathrm{GmbH}$, Dessau, Germany) was supplied in the appropriate particle size and was therefore only sieved to select particles $\leq 150 \mu \mathrm{m}$.

\section{Microorganisms and culture conditions}

S. cerevisiae $\mathrm{H} 205$ was provided ready for use by Deutsche Hefewerke GmbH (Nürnberg, Germany).

M. brunneum strain CB15 was obtained from Prof. Dr. Stefan Vidal (Agricultural Entomology, Department for Crop Science, Georg-August-University Goettingen, Germany). To produce aerial conidia (AC) used for inoculation of submerged cultures, freeze-dried biomass was thawed in Potato Dextrose Broth (PDB) and cultivated on Potato Dextrose Agar (PDA) in the dark at $25^{\circ} \mathrm{C}$ for 7 days. Plates were sealed with Parafilm M (Pechiney Plastic Packaging Inc., IL, USA) to avoid moisture loss. Then, the pre-culture was inoculated with $\mathrm{AC}$ resulting in a final concentration of $1 \times 10^{6} \mathrm{AC} / \mathrm{mL}$ and cultivated in liquid medium modified according to Krell et al. (2018) (7.5\% glucose, 4.0\% ANiPept (ANiMOX GmbH, Berlin, Germany, batch No. 1176), $7.0 \%$ polyethylene glycol 200 ) at $25^{\circ} \mathrm{C}$ and $150 \mathrm{rpm}$ in $250 \mathrm{~mL}$ baffled shake flasks for $48 \mathrm{~h}$. Cultures were inoculated with $5.0 \%(\mathrm{v} / \mathrm{v})$ vegetative pre-culture and cultivated for $72 \mathrm{~h}$. The mycelial biomass was harvested by vacuum filtration through filter paper (Whatman No. 4, qualitative, $20-25 \mu \mathrm{m}$ pore size) and rinsed with $0.9 \% \mathrm{NaCl}$ solution to remove blastospores and residual cultivation medium.

\section{Bead production}

The preparation of calcium alginate beads was carried out with sterilized materials under aseptic conditions. Prior to encapsulation, sodium alginate (CEROGA Sodium Alginate Type NA5030, C.E. Roeper GmbH, Hamburg, Germany) was dissolved in ultrapure water to a final concentration of
$4.0 \%$ and autoclaved for $6 \mathrm{~min}$ at $121^{\circ} \mathrm{C}$. The encapsulation suspensions were prepared as follows.

\section{Calcium alginate/starch beads}

The encapsulation suspension for the control beads was produced by thoroughly mixing $2.0 \%$ calcium alginate with $20.0 \%$ native corn starch (CIF GmbH, Siegburg, Germany) for $10 \mathrm{~min}$ until suspended. Finally, water was added to reach final concentrations.

Encapsulation suspension with casein additives was produced by mixing $2.0 \%$ sodium alginate with $13.3 \%$ native corn starch for $5 \mathrm{~min}$ and then with $6.7 \%$ either casein or formalin-casein for 5 min until suspended. Because the suspension became viscous, the water required to reach final concentrations was added shortly before dripping.

\section{Kill beads}

The encapsulation suspension for the control Kill beads was produced by mixing $2.0 \%$ sodium alginate with $20.0 \%$ native corn starch for 10 min until suspended. Then, $1.0 \%$ M. brunneum mycelial biomass was added and gently stirred for $2 \mathrm{~min}$. Water was added to reach the final concentrations.

The encapsulation suspension with either casein or FC was produced by mixing $2 \%$ sodium alginate with $13.3 \%$ native corn starch for $5 \mathrm{~min}$, and subsequently with $6.7 \%$ either casein or formalin-casein for 5 min until suspended. Then, $1.0 \%$ M. brunneum mycelial biomass was added and gently stirred for $2 \mathrm{~min}$. The water required to reach final concentrations was added shortly before dripping.

\section{Attract-and-kill beads}

The encapsulation suspension for the control A\&K beads was produced by mixing $2.0 \%$ sodium alginate with $16.7 \%$ S. cerevisiae for $5 \mathrm{~min}$ until blended and then with $20.0 \%$ native corn starch for $10 \mathrm{~min}$. Then, $1.0 \%$ M. brunneum mycelial biomass was added and gently stirred for $2 \mathrm{~min}$. Finally, water was added to reach final concentrations.

The encapsulation suspensions with casein or FC were prepared the same way, but only $13.3 \%$ native corn starch was mixed for 5 min before $6.7 \%$ either casein or FC was added and mixed for another $5 \mathrm{~min}$.

\section{Bead formation}

Beads were prepared by dripping the suspension into a sterile $180 \mathrm{mM} \mathrm{CaCl}{ }_{2}$ solution by means of a single syringe pump (Cole-Parmer, Vernon Hills, USA), with a $0.9 \mathrm{~mm}$ cannula (Sterican, B. Braun Melsungen AG, Melsungen, Germany). The pumping speed was set to $4 \mathrm{~mL} / \mathrm{min}$. After $20 \mathrm{~min}$, beads were separated and washed with ultrapure 
water. Moist beads were dried under a laminar flow at room temperature $\left(23^{\circ} \mathrm{C}, 30 \%\right.$ relative humidity) for $24 \mathrm{~h}$. Alginate/starch beads were subsequently dried over silica to reach a final water activity of 0.2 , unless specified otherwise. Water activities were controlled with a water activity meter (LabMASTER-aw, Novasina AG, Lachen, Switzerland) at $25{ }^{\circ} \mathrm{C}$.

\section{Analysis of calcium alginate/starch beads}

\section{Determination of $\mathrm{C} / \mathrm{N}$ ratio}

The $\mathrm{C} / \mathrm{N}$ ratio of alginate beads was analyzed with a $\mathrm{C} / \mathrm{N}$ elemental analyzer (Unicube ${ }^{\circledR}$, temperature programmed desorption column, Elementar, Langenselbold, Germany) to assess the nutritive influence of casein and FC. Dried calcium alginate beads with starch or starch and either casein or FC were grinded with a ball mill to obtain a homogenous composition of the sample matrix. The amount of $5 \mathrm{~g}$ of each matrix was weighed in tin containers and loaded into an automatic sampler. Each sample matrix was measured twice.

\section{Determination of bead size, bead count and bead shape}

The particle projected area diameters derived from digital images of the beads were determined using the open source image processing program Fiji (ImageJ, U. S. National Institutes of Health, Bethesda, USA) (Schindelin et al. 2012). For this, beads were placed in the center of a Petri dish on a black background and images including a reference scale were taken using a tripod. For following batch analysis by help of a macro (Online Resource 1, Fig. A1), images were taken at the same magnification. The parameters of the macro were adapted for the respective batch. The determined diameters were automatically exported in an excel file and used to calculate the bead surface area. Beads were counted simultaneously to bead size measurements. Additionally, the bead shape of dried and rehydrated beads (after $48 \mathrm{~h}$ ) was determined, which is represented by the circularity and the sphericity factor of the particle projected area. The latter was determined according to Chan et al. (2011).

Sphericity factor $=\frac{d_{\max }-d_{\min }}{d_{\max }+d_{\min }}$

where $d_{\text {max }}$ is the largest diameter and $d_{\text {min }}$ is the smallest diameter perpendicular to $d_{\max }$.

\section{Determination of water absorption and water activity}

Rehydration was measured as a function of time according to Vreeker et al. (2008).
In preliminary investigations we found that the rehydration of beads reaches its maximum within $48 \mathrm{~h}$. Based on these findings, we chose to examine the rehydration within this period more closely. Therefore, the water absorption of beads was determined gravimetrically in defined time intervals between 0 min and $48 \mathrm{~h}$.

Approximately 100 dried beads were placed on $15 \mathrm{~mL}$ $1.5 \%$ water agar in closed Petri dishes. For each time point separate Petri dishes were prepared in three replicates. The exact number of beads of each sample was determined by digital image analysis using the open source image processing program Fiji (see 'Determination of bead size, bead count and bead shape') and was later set in relation to the measured sample weight.

Beads were withdrawn from water agar and excess water was carefully removed by blotting on lint-free tissue before weighing on a precision balance (VWR, Pennsylvania, USA) in small, closed Petri dishes. Rehydration $R(t)$ is expressed as

$R(t)=\frac{m(t)-m_{d}}{m_{0}-m_{d}}$

where $m(t)$ represents the mass of beads during rehydration; $m_{0}$ is the mass of fresh beads before drying and $m_{d}$ is the mass of dried beads.

Afterwards the samples were transferred into special airtight containers for water activity measurements and stored until measured. Water activities were determined with a water activity meter (LabMASTER-aw, Novasina AG, Lachen, Switzerland) at $25^{\circ} \mathrm{C}$. Water activity and temperature stable time were set to $2 \mathrm{~min}$ and $1 \mathrm{~min}$, respectively.

\section{Determination of bead density}

The bead density $\rho_{p}$ of the different calcium alginate/ starch formulations with either casein or FC was calculated using the settling velocity $(v)$ in water according to Stokes equation.

$v=\frac{\left(\rho_{p}-\rho_{f}\right) \cdot \bar{d}^{2} \cdot g}{18 \cdot \eta}$

where $\rho_{f}$ is the density of water, $d$ is the mean bead projected area diameter, which was determined for 50 beads (see 'Determination of bead size, bead count and bead shape'), $g$ is the gravity with $9.81 \mathrm{~m} / \mathrm{s}, \eta$ represents the dynamic viscosity of water and is $0.000931 \mathrm{~kg} / \mathrm{m} \mathrm{s}$. Digital video recordings of the beads at $120 \mathrm{fps}$ were taken and the settling velocity was determined with an open source video editing program (VideoPad Video Editor Free, NCH Software). For each kind of formulation 20 beads were analyzed. 


\section{Determination of pore volume distribution and pore size}

We used differential scanning calorimetry (DSC) to assess the pore volume distributions and therefore how casein or $\mathrm{FC}$ influence the pore sizes in calcium alginate/starch beads. DSC measurements were modified according to the measurements of pore sizes in silica gels by Kazuhiko Ishikiriyama et al. (1995a, b) and Maloney (2000) using a Mettler Toledo DSC-3 device.

Dried beads were rehydrated on water agar in Petri dishes and then immersed in excessive water overnight to remove residual ions and to fill the pores with water. Accurately weighed samples (3-6 mg) were sealed in $40 \mu \mathrm{L}$ aluminum pans with pins. Because of their weight and size, rehydrated beads needed to be cut to fit into the aluminum pans. The samples were measured against an empty reference pan. Nitrogen served as purge gas at $50 \mathrm{ml} / \mathrm{min}$. The measurements were performed over a temperature range from 233.15 to $283.15 \mathrm{~K}$ and melting curves were measured to determine the pore volume distributions. Samples were rapidly cooled to $233.15 \mathrm{~K}$, held isothermally for $30 \mathrm{~min}$, and subsequently heated to $183.15 \mathrm{~K}$ at a low scanning rate of $0.5 \mathrm{~K} / \mathrm{min}$ to avoid thermal and time delays. After DSC measurements, samples were dried at $60{ }^{\circ} \mathrm{C}$ for 3 days and the dry weight or rather the mass of the water was determined. Measurements were conducted three times with three different beads for each kind of formulation. Resulting peaks were analyzed according to Maloney (2000) with the STARe evaluation software (Version 15, Mettler Toledo, Columbus, USA) and transformed into pore volume distributions using Microsoft Excel (Microsoft Corporation, Washington, USA). The relationship between the pore diameter $D$ and the melting point depression $\Delta T_{m}$ is described by the Gibbs -Thomson equation:

$D=\frac{4 \cdot V_{m} \cdot T \cdot \sigma_{l s}}{\Delta H_{m} \cdot \Delta T_{m}}$

$V_{m}$ is the mol volume of the used absorbate, in this case, water with $19.610^{-6} \mathrm{~m}^{3} / \mathrm{mol}, T$ is the melting point of water $273.14 \mathrm{~K}, \sigma_{l s}$ represents the calcium alginate and water boundary tension, which was previously determined by contact angle measurement using the sessile drop technique (OCA 15EC model, SCA 20 control software module, DataPhysics Instruments GmbH, Filderstadt, Germany) and resulted in $78.44 \mathrm{mN} / \mathrm{m} . \Delta H_{m}$ is the heat of fusion of water which is $6.02 \mathrm{~kJ} / \mathrm{mol}$.

The total volume $V$ of the pores with the relevant diameter can be determined from the peak area (heat fusion $H_{I}$ ) measured at the according temperature

$V=\frac{H_{I}}{\left(\rho_{w} \cdot \Delta H_{s}\right)}$ $\rho_{w}$ represents the density of water or ice at the corresponding temperature. $\Delta H_{s}$ is the temperature dependent specific heat of fusion of the ice-water transition concerning freezable pore water which was determined according to Ishikiriyama et al. (1995a, b) using the equation of Randall (National research council of the United States of America 1930) in $\mathrm{J} / \mathrm{g}$ where $\Delta T_{m}$ is the depression of the melting temperature of water.

$\Delta H_{s}=334.1+2.119 \cdot \Delta T-0.00783 \cdot \Delta T$

The layer thickness of the non-freezable water was neglected.

\section{Assessment of the fungal activities}

\section{Determination of the spore density on beads}

The spore forming activity of M. brunneum on Kill and A\& $\mathrm{K}$ beads was determined by counting the conidia formed on the bead surface using a cell counting chamber (Thoma new, Paul Marienfeld GmbH \& Co. KG, Lauda-Königshofen, Germany).

Beads were produced as described previously (see 'Bead production') with biomass from the same culture. Dried beads were rehydrated and eventually incubated on $1.5 \%$ water agar in sealed Petri dishes at $25^{\circ} \mathrm{C}$ in the dark. The spore density was determined in defined time intervals within 14 days. At each sampling point and for each kind of formulation, ten beads were analyzed. Each bead was transferred into $1 \mathrm{~mL} 0.1 \%$ Tween 80 and thoroughly mixed (Vortex Genie, neoLab Migge GmbH, Heidelberg, Germany) for at least $1 \mathrm{~min}$ to detach the spores. To calculate the spore density, the bead surface area was determined from the projected area diameter (see 'Determination of bead size, bead count and bead shape') of 100 rehydrated beads per formulation.

The spore density was evaluated again after 21 days, in order to compare the sporulation with that in a vented system (see ' $\mathrm{CO}_{2}$ release measurements from beads'). It should be noted that over time mycelial growth was favored and spore densities may be underestimated, since spores adhered in the dense mycelium.

\section{$\mathrm{CO}_{2}$ release measurements from beads}

To assess how casein and FC influenced the metabolic activity of S. cerevisiae and/or M. brunneum, the amount of $\mathrm{CO}_{2}$ released from dried and rehydrated beads was measured using a carbon dioxide meter with pump aspirated sampling (CARBOCAP® GM 70; Vaisala Oyj, Helsinki, Finland).

For this purpose, $1 \mathrm{~g}$ of dried alginate/starch beads, Kill beads or A\&K beads with or without either casein or FC 
were rehydrated and eventually incubated at room temperature in the dark on $5 \mathrm{~mL}$ water agar in sterile glass bottles with a defined volume of $134.4 \mathrm{~mL}$. The glass bottles were vented with sterile pressured air to avoid $\mathrm{CO}_{2}$ accumulation and inhibition during cultivation. Additionally, the pressured air was humified using a gas washing bottle to prevent the beads and the water agar from drying. The relative humidity reached approximately $80 \%$. By means of a compressed air distributor, 18 vessels were vented and cultured at once (Fig. 6a). The air flow rate was set to $15 \mathrm{~L} / \mathrm{h}$ (equals one volume exchange per $10 \mathrm{~min}$ ) using a variable area flowmeter.

In order to determine the $\mathrm{CO}_{2}$ productivity, the initial $\mathrm{CO}_{2}$ concentration was measured before the glass bottles were closed for $60 \mathrm{~min}$. The in- and outlets of the carbon dioxide meter pump were connected to the glass bottles via sterile filters, thus the air circulated during the measurement. Each sample was measured for $2 \mathrm{~min}$. Subsequently, the device was conditioned with ambient air for at least $1 \mathrm{~min}$. The amount of released $\mathrm{CO}_{2}$ was measured every day over a period of 20 days and was converted from ppm to $\mathrm{mL} \mathrm{CO}_{2}$ produced per $\mathrm{g}$ dried beads in $1 \mathrm{~h}$. All measurements were performed in three replicates (three glass bottles) simultaneously. The biomass from the same culture or batch was used for each kind of formulation. The spore density on beads was determined after the experiment was terminated, i.e. after 21 days.

The $\mathrm{CO}_{2}$ productivity of control alginate/starch beads without M. brunneum or S. cerevisiae biomass, was previously evaluated and was negligible low (Online Resource 1, Fig. A2) and therefore, in subsequent experiments neglected. Due to surprising results, the experiment was repeated for Kill beads and A\&K beads with and without FC.

\section{Statistical analysis}

Statistical analyses were conducted with SPSS Statistics v. 27 (SPSS, Chicago, USA) with a significance level of $\mathrm{p}<0.05$, except for spore density analysis of sealed and vented cultures, which were conducted with a significance level of $\mathrm{p}<0.01$. All data are presented as mean values \pm standard deviations (SD). Percentage data on the water activity and the rehydration factor were arcsine transformed prior to analysis of normality and homogeneity of variance with Shapiro-Wilk's and Levene's test, respectively. To analyze the influence of the treatments or the influence of treatment and time, one-way or repeated measures (RM) analysis of variance (ANOVA) with Bonferroni post hoc tests were conducted for normally distributed and homogeneous data. Welch's correction was applied for nonhomogeneous variances. The sphericity of the matrix assumption was assessed with the Mauchley sphericity test and F values were corrected accordingly using the Greenhouse-Geisser approach.
For non-normally distributed data sets, Kruskal-Wallis tests with multiple comparisons between groups were calculated.

\section{Results}

\section{Analysis of calcium alginate/starch beads}

First, we investigated plain calcium alginate/starch beads without any fungal biomass in terms of their nutrient content, water absorbency and pore structure.

Beads of different compositions appeared relatively homogeneous, rigid and did not disintegrate. The sphericity factor of dried and rehydrated beads were as follows, $0.076 \pm 0.010$ and $0.082 \pm 0.003$ for alginate/starch beads, respectively, $0.066 \pm 0.003$ and $0.071 \pm 0.008$ with $\mathrm{FC}$, respectively, and $0.072 \pm 0.010$ and $0.065 \pm 0.001$ with casein, respectively (three replicates with $89-112$ beads per replicate). The circularity of beads of different composition, either dried or rehydrated, was consistently between $0.88-0.89$.

\section{Increased $\mathrm{C} / \mathrm{N}$ ratio by formalin-casein}

We analyzed the formulation matrices with a $\mathrm{C} / \mathrm{N}$ elemental analyzer to assess the nutritional influence of casein and FC. As expected, alginate/starch beads contained almost no nitrogen. Despite equal proportions of casein and FC in the mixture, incorporating FC, however, doubled the nitrogen content compared to casein, resulting in higher $\mathrm{C} / \mathrm{N}$ ratios of 17.33 compared to 9.72 , respectively (Table 1 ). Measurements were repeated once (Online Resource 1, Table A1).

In a simple cultivation test on agar with starch and either casein or FC, we observed slightly enhanced mycelial growth for FC (Online Resource 1, Fig. A3).

\section{Enhanced rehydration by formalin-casein}

In order to study whether casein or FC influence the water absorption and water activity of alginate/starch beads, dried beads were rehydrated on water agar over a period of $48 \mathrm{~h}$.

Alginate/starch beads without or with either casein or FC were dried to $\mathrm{a}_{\mathrm{w}} 0.174-0.175,0.239-0.256$ and $0.165-0.178$, respectively. The water absorption of beads with either casein or FC differed significantly, also across time $\left(\mathrm{F}_{2,6}=7.3, P=0.03, \mathrm{~F}_{3.2,9.8}=5.3, P=0.02\right.$, respectively). Interestingly, casein initially decelerated the water absorption compared to alginate/starch beads with or without FC, finally reaching a rehydration factor of alginate/ starch beads only (Fig. 1). In contrast, FC accelerated the water absorption after 15 min compared to alginate/starch, thus, resulting in a higher maximum rehydration factor of $0.37 \pm 0.01$ compared to $0.27 \pm 0.00$, respectively. 


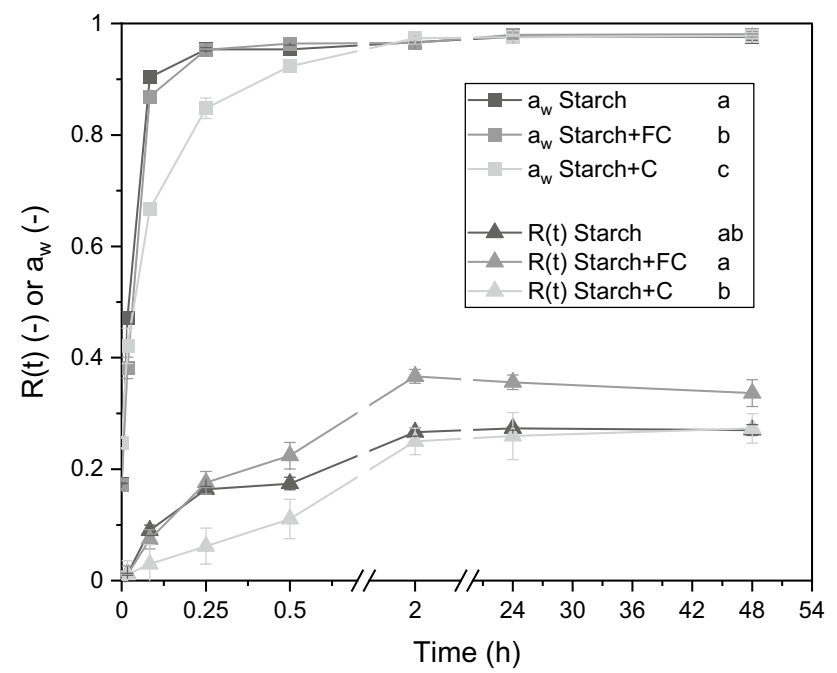

Fig. 1 The maximum water absorption but not the water activity of alginate/starch beads was enhanced by formalin-casein (FC). The rehydration factor and the water activity of alginate/starch beads containing casein (C) or FC were rehydrated on $1.5 \%$ water agar at room temperature. Different letters next to the legend description indicate significant differences according to RM-ANOVA with Bonferroni post-hoc test at $\mathrm{P}<0.05(\mathrm{n}=3)$

The bead composition and the interaction of bead composition and time also affected the water activity significantly $\left(\mathrm{F}_{2,6}=256.3, P<0.001, \mathrm{~F}_{5.4,16.4}=59.9, P<0.001\right.$, respectively). Casein initially decreased water activity of beads, reaching only $0.848 \pm 0.019$ after $15 \mathrm{~min}$, compared to $0.953 \pm 0.007$ for FC and $0.954 \pm 0.003$ for alginate/starch. Regarding rehydration factor or water activity, all formulations reached equilibrium after $2 \mathrm{~h}$.

Overall, casein, initially decelerated the water absorption, and thus reached the equilibrium of starch only. FC, however, partly accelerated and finally enhanced the water absorption of alginate/starch beads.

\section{Altered bead porosity and bead density}

Next we investigated how casein and FC affect the internal, porous structure of the alginate/starch beads, more specifically, bead density, pore volume and pore size. The bead density of rehydrated beads was determined via settling velocity in water, and the pore volume as well as the pore size were determined by thermoporometry using differential scanning calorimetry.

The formulation composition significantly affected the bead density $\left(\mathrm{F}_{2,59}=127.7, P<0.001\right)$ and the median pore size $\left(\mathrm{F}_{2,9}=7.2, P=0.03\right)$, but not the total pore volume $\left(\mathrm{F}_{2,9}=4.62, P=0.10\right)$.

The pore volume distributions revealed a broader spectrum of pores for FC with more pronounced shoulders and peaks were visibly shifted to larger pore sizes for both

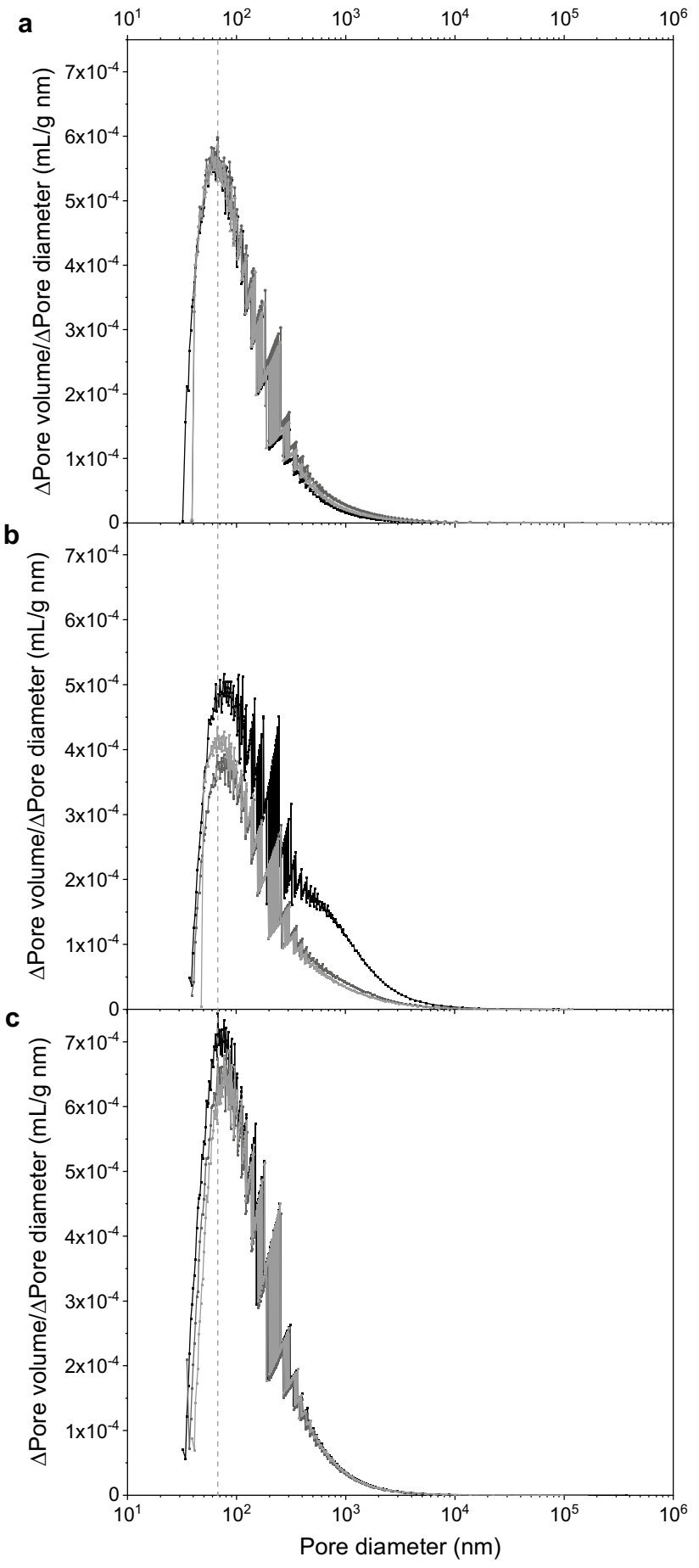

Fig. 2 The pore volume distributions of rehydrated alginate/starch beads (a) are shifted when either formalin-casein (b) or casein (c) is added, and are broadened in case of formalin-casein. Distributions were determined by differential scanning calorimetry. Replicates $(n=3)$ are shown in different grey values. The dashed line marks the peak of alginate/starch beads

casein and FC (Fig. 2). Consequently, both casein and FC increased the median pore diameter by 19 and $31 \%$, respectively (Fig. 3). The results on pore volume, determined by 
Table 1 Elemental analysis of alginate/starch beads and alginate/ starch beads with either casein or formalin-casein (FC) by means of an elemental analyzer

\begin{tabular}{lllc}
\hline Formulation & Carbon (\%) & Nitrogen (\%) & C/N ratio (-) \\
\hline Alginate/starch & 37.05 & 0.03 & 1402.25 \\
$\begin{array}{l}\text { Alginate/starch with } \\
\quad \text { casein }\end{array}$ & 37.93 & 2.19 & 17.33 \\
Alginate/starch with FC & 40.65 & 4.18 & 9.72 \\
\hline
\end{tabular}

thermoporometry, were not significant, but there was a slight trend towards larger pore volume with FC, which is supported by the findings on bead density. In contrast to casein, FC lowered the bead density of alginate/starch beads
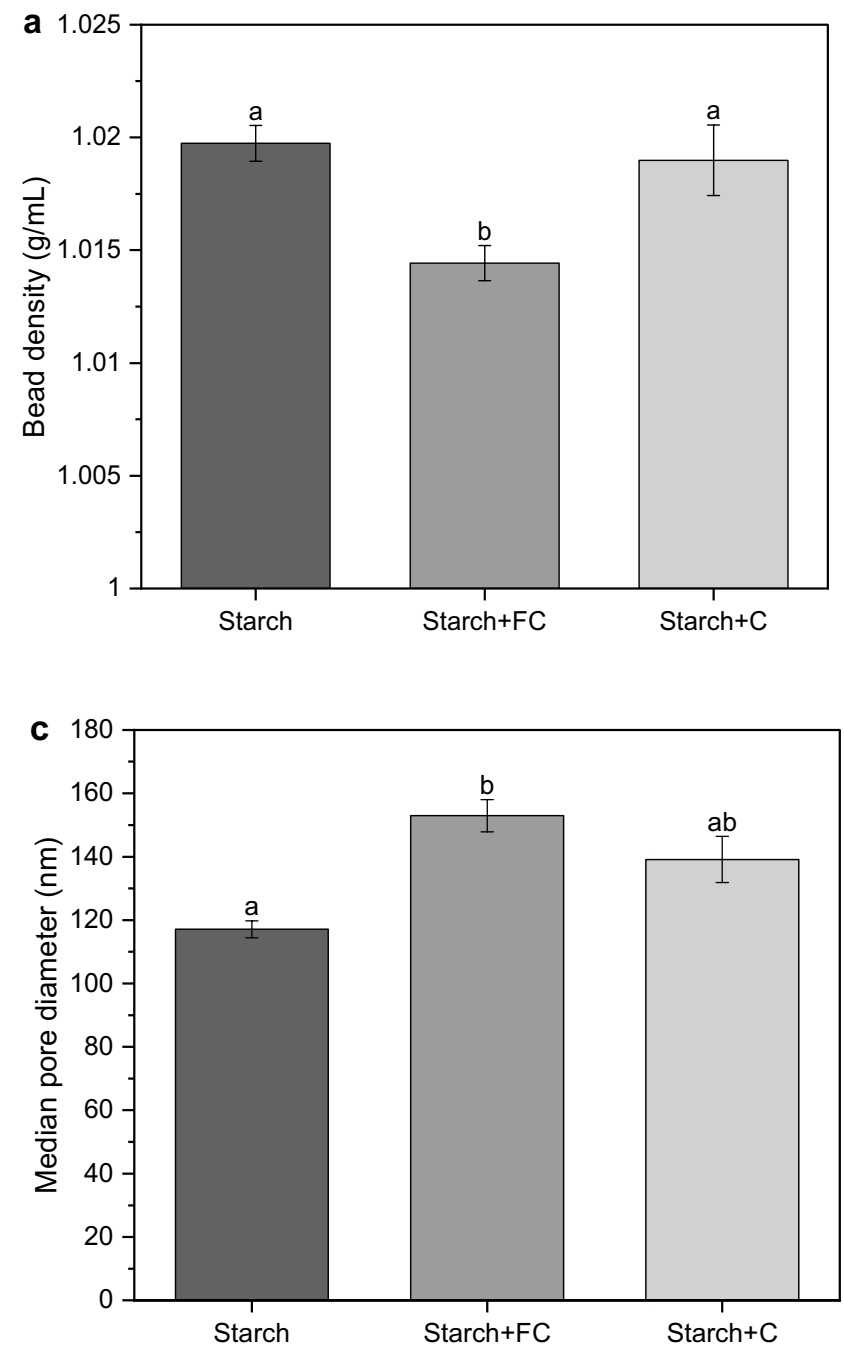

Fig. 3 Incorporating formalin-casein (FC) into alginate/starch beads reduced the bead density (a) and increased the total pore volume (b) as well as the median pore diameter (c). The relationship of these three parameters becomes clear in $\mathbf{d}$. Alginate/starch beads and alginate/starch beads with either FC or casein were rehydrated prior to significantly from $1.02 \pm 3.110^{-7} \mathrm{~g} / \mathrm{mL}$ to $1.014 \pm 2,810^{-7} \mathrm{~g} /$ $\mathrm{mL}\left(\mathrm{F}_{2,57}=127.753, P<0.001\right)$. Additional data are given in Online Resource 1 Table A2.

In sum, FC had a greater impact on porosity and density than casein, resulting in less dense beads with larger pores.

\section{Assessment of the fungal activity}

Next, we assessed the activity of either M. brunneum, or both $S$. cerevisiae and M. brunneum, in Kill beads or A\&K beads, respectively, by determining the spore formation and $\mathrm{CO}_{2}$ release.
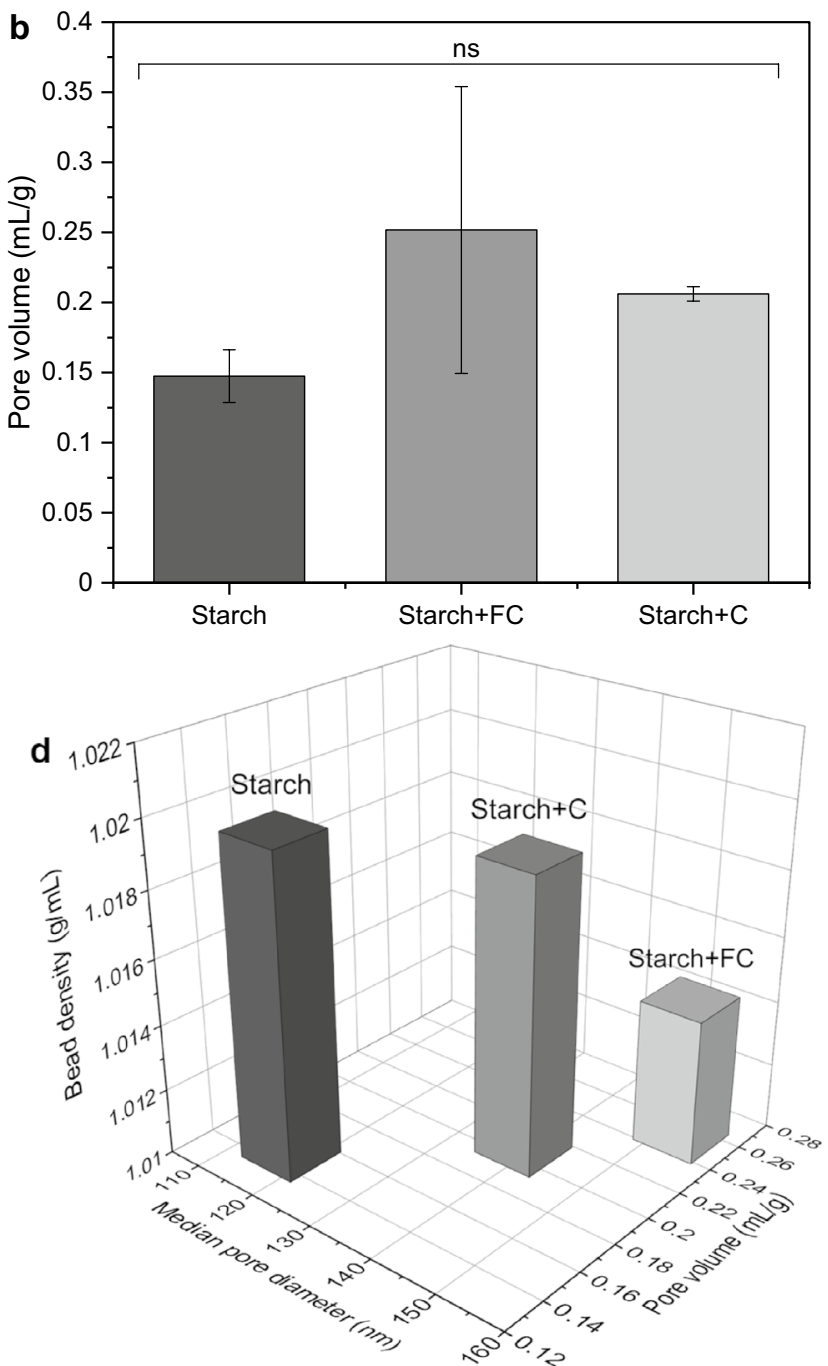

analysis. Different letters above bars indicate significant differences according to ANOVA with Bonferroni post-hoc test at $\mathrm{P}<0.05$ for bead density $(n=20)$ and Kruskal-Wallis test at $\mathrm{P}<0.05$ for pore volume and median pore diameter $(n=3)$ 


\section{Enhanced sporulation of Metarhizium brunneum}

We asked, whether casein or FC impact the spore formation of $M$. brunneum on beads. Moreover, it should be investigated to what extent a possible effect can be observed on beads containing additionally encapsulated $S$. cerevisiae. Therefore M. brunneum was individually encapsulated (Kill beads) or co-encapsulated with $S$. cerevisiae (A\&K beads) in alginate/starch beads with either casein or FC. Beads were dried to $\mathrm{a}_{\mathrm{w}}$ 0.29-0.46. Both casein and FC increased spore density on Kill and A\&K beads, but only FC led to a significant difference on both $\left(\mathrm{F}_{2,27}=4.0, P=0.03 ; \mathrm{F}_{2,27}=116.4\right.$, $P<0.001)$ and also across time $\left(\mathrm{F}_{2,27}=4.3, P=0.02\right.$; $\mathrm{F}_{3.6,48.3}=28.7, P<0.001$ ) (Fig. 4). Accordingly, maximum spore density on Kill and A\&K beads was enhanced by FC by the factor 7 and 3, respectively. Casein, however, showed strong differences between replicas. On the one hand, strong mycelial growth and sporulation was observed on the beads, but on the other hand, there were beads with almost no mycelial growth or even excessive yeast growth.
After 7 days, the spore forming rate of M. brunneum was particularly enhanced by FC, irrespective of whether $S$. cerevisiae was co-encapsulated. Incorporating FC did not accelerate the onset of spore formation but increased the spore density on both Kill-beads and A\&K beads. Nevertheless, the impact was more pronounced when $M$. brunneum was encapsulated individually. Overall, $\mathrm{FC}$ had a higher impact than casein, since incorporating casein showed inconsistent results.

Increasing the FC content and omitting starch concurrently, did not increase or fasten the spore formation, in contrast, it significantly reduced the spore density over time, presumably due to the lack of carbon (Online Resource 1, Fig. A4).

For studies on $\mathrm{CO}_{2}$ release (see ' $\mathrm{CO}_{2}$ release from beads') the different Kill and $\mathrm{A} \& \mathrm{~K}$ formulations from the same batch previously described, were aerated during cultivation. The sporulation differed quite obviously in the course of the experiment and it became apparent that to some extent spores were formed earlier (Fig. 5). Therefore, the question
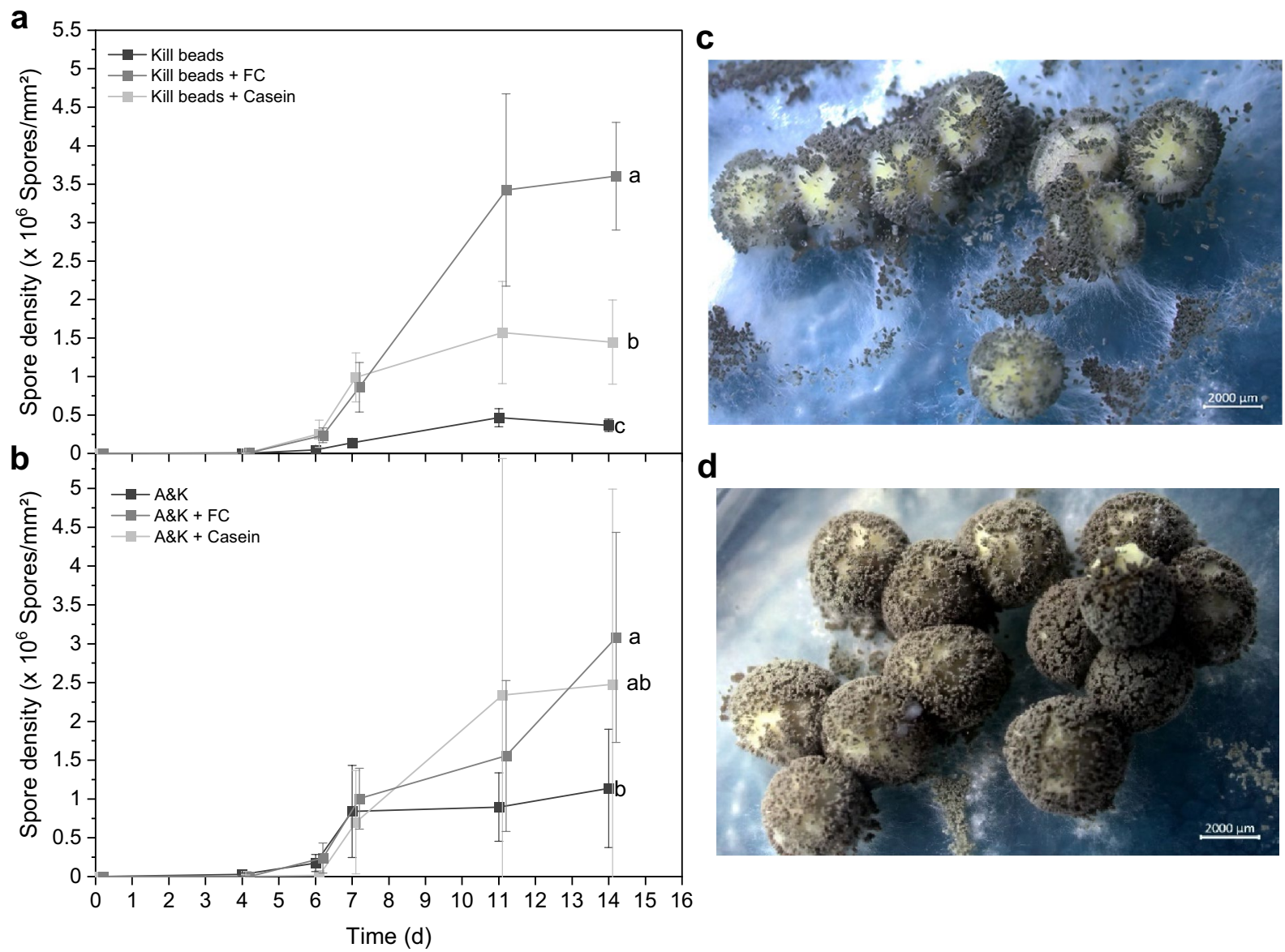

d

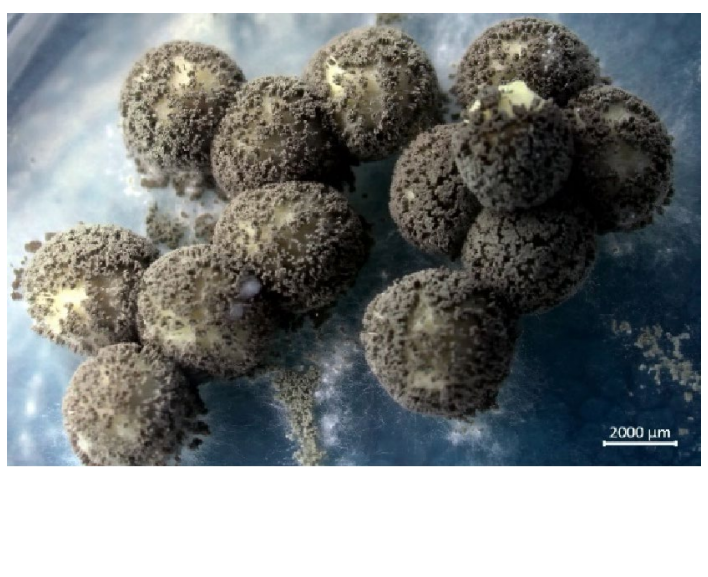

Fig. 4 Formalin-casein (FC) significantly enhanced the spore formation on both Kill beads (a) and A\&K beads (b) compared to beads containing only starch and even compared to Kill beads containing casein. Beads were rehydrated and cultivated on $1.5 \%$ water agar at
$25{ }^{\circ} \mathrm{C}$. Different letters indicate significant differences according to RM-ANOVA with Bonferroni Post hoc test $(n=10)$. Spore density on A\&K beads (c) was visibly enhanced by formalin-casein (d) after 17 d 

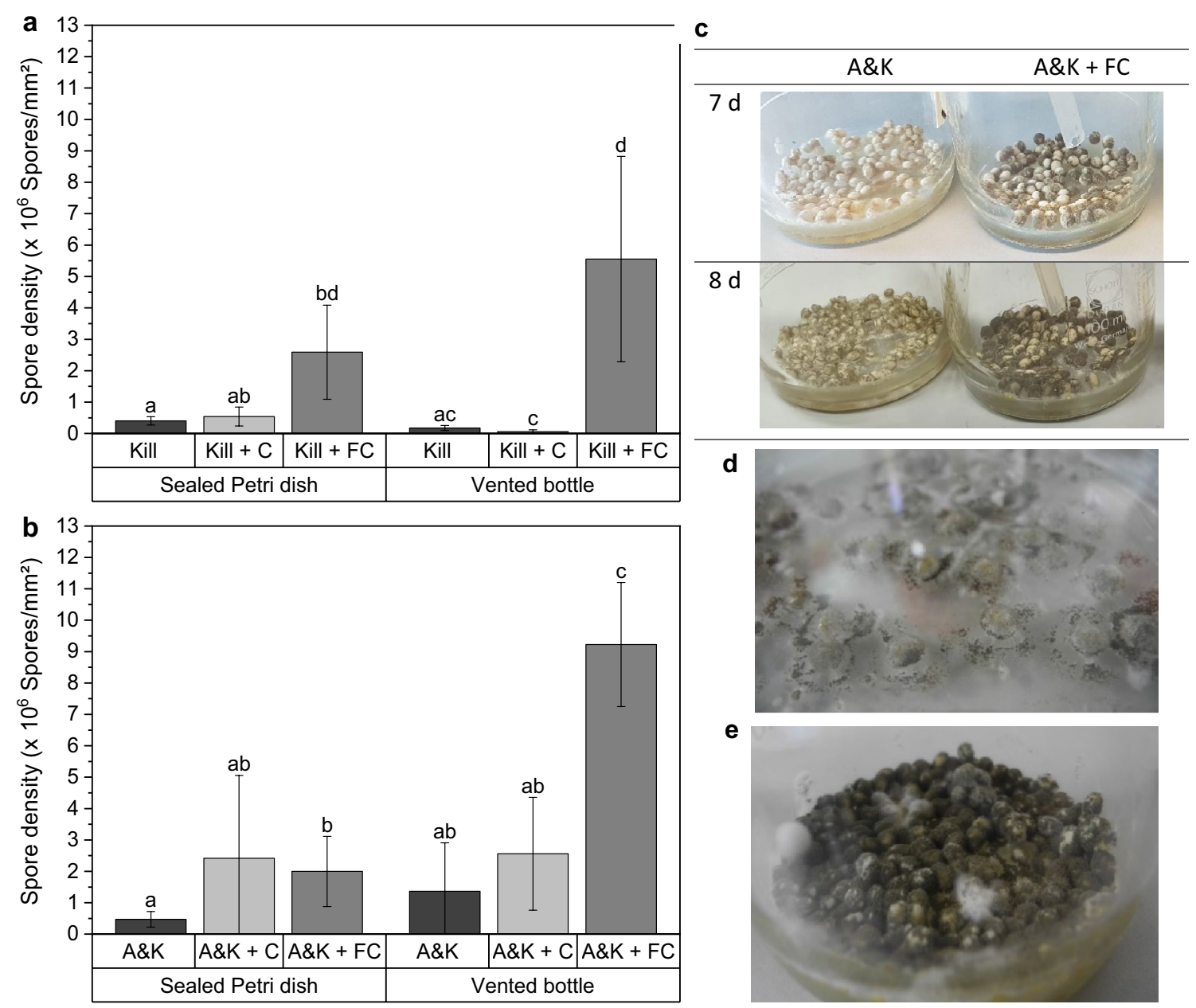

Spore formation was visibly accelerated on A\&K beads by FC when

Fig. 5 Aeration significantly increased spore density exclusively when formalin-casein (FC) is incorporated in Kill beads (a) or A\&K beads (b). Beads were rehydrated and cultivated on $1.5 \%$ water agar either in sealed Petri dishes at $25{ }^{\circ} \mathrm{C}$, or in vented glass bottles at room temperature. Different letters above bars indicate significant differences according to Kruskal-Wallis-test with $\mathrm{p}<0.01 \quad(\mathrm{n}=10)$.

arose, whether there is an interaction between aeration and the formulation additives. Thus, spore densities of beads cultured in sealed Petri dishes or in vented glass bottles were determined after 21 days.

By aeration, the spore density was increased on both Kill beads and A\&K beads but only when FC was incorporated (Fig. 5). As a result, the spore density on A\&K beads with FC was significantly increased more than fourfold from $(2.0 \pm 1.1) \cdot 10^{6}$ spores $/ \mathrm{mm}^{2}$ to $(9.2 \pm 2.0) \cdot 10^{6}$ spores $/ \mathrm{mm}^{2}$ $\left(\mathrm{H}_{5}=51.509, P<0.001\right)$.

Taken together, incorporating FC and subsequently venting the cultures increased the spore density even by the factor 18. Surprisingly, aeration had a negative impact on spore formation on Kill beads when casein was added (from $5.4 \pm 3.010^{5}$ to $0.5 \pm 0.510^{5}$ spores $/ \mathrm{mm}^{2}$ ). However, this was not seen for $\mathrm{A} \& \mathrm{~K}$ beads, confirming the inconsistency rehydrated and cultivated in vented glass bottles, as can be seen after 7 and 8 days (c). Compared to the cultivation in sealed Petri dishes (d), aeration visibly enhanced spore yield of A\&K beads containing FC (e)

of results mentioned previously. Overall, enhanced mycelial growth could be observed to some extent, but a positive and significant change in sporulation due to aeration was observed exclusively for FC. These results were in line with the drying kinetics of alginate/starch beads, which showed faster drying when FC was incorporated (Online Resource 1, Fig. A5).

\section{Enhanced $\mathrm{CO}_{2}$ release from beads}

To evaluate how casein and FC influence the respiratory activity of $M$. brunneum and $S$. cerevisiae and hence the $\mathrm{CO}_{2}$ release from beads, two different bead formulations namely Kill beads containing only M. brunneum and A\&K beads containing both fungi, M. brunneum and S. cerevisiae, were examined. 


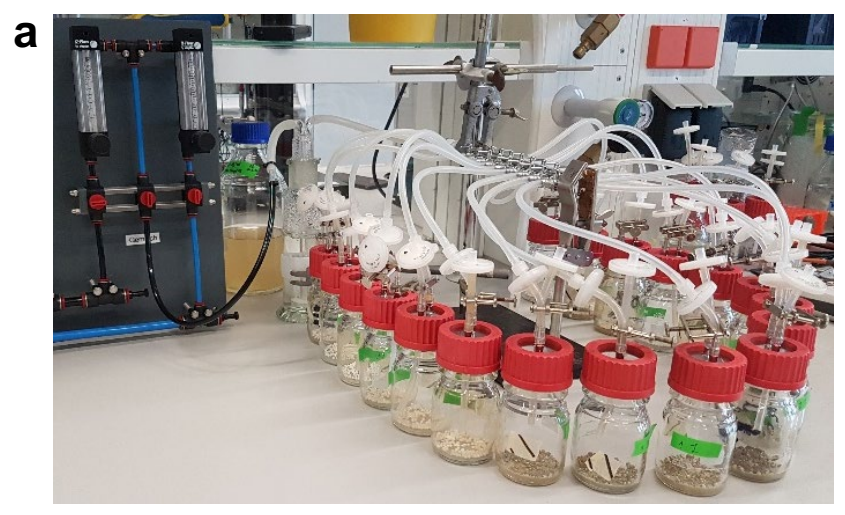

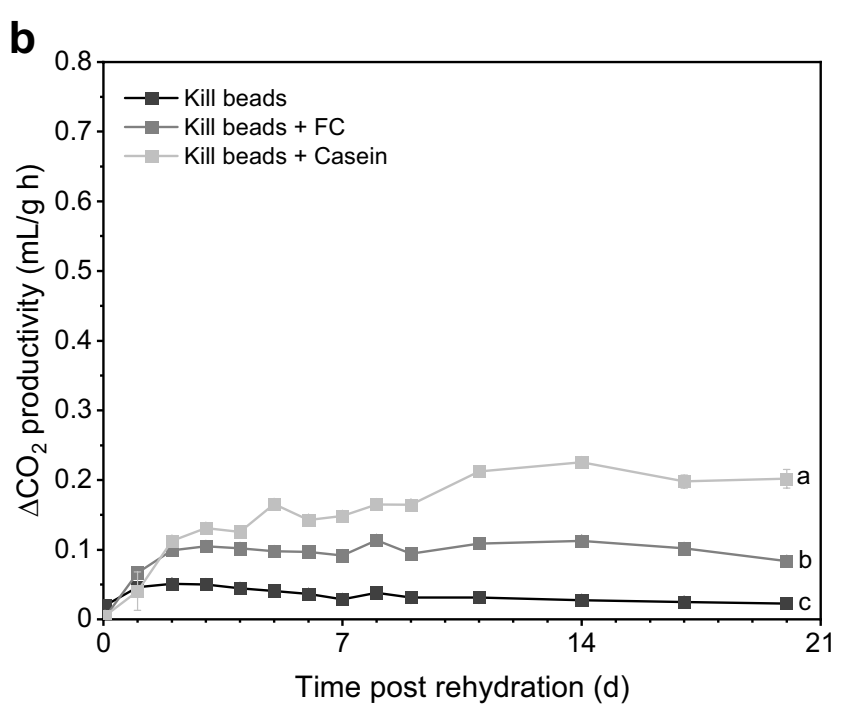

Fig. 6 Experimental set-up (a) for $\mathrm{CO}_{2}$ release measurements. Beads were rehydrated and cultivated on $1.5 \%$ water agar at room temperature in vented glass bottles at $15 \mathrm{~L} / \mathrm{h}$ (one volume exchange per $10 \mathrm{~min}$ ) using a variable area flowmeter and a compressed air distributor. Formalin-casein (FC) significantly increased the $\mathrm{CO}_{2}$ productiv-

The bead composition significantly influenced the $\mathrm{CO}_{2}$ productivity of both Kill and A\&K beads $\left(\mathrm{F}_{2,6}=1708.9\right.$, $P<0.001$ and $\mathrm{F}_{2,6}=271.7, P<0.001$, respectively, Fig. 6). Accordingly, the Kill beads $\mathrm{CO}_{2}$ production increased twofold within the first 2 days with either casein or FC compared to plain Kill beads. Over the course of 5 days a greater influence by casein on the Kill beads was revealed, resulting in a doubled and even tenfold $\mathrm{CO}_{2}$ productivity compared to Kill beads with or without FC after 10 days. The total amount of $\mathrm{CO}_{2}$ produced by $M$. brunneum was five-folded from $16 \mathrm{~g} / \mathrm{L}$ to $81 \mathrm{~g} / \mathrm{L}$ by casein and three-folded to $47 \mathrm{~g} / \mathrm{L}$ by FC.

Plain A\&K beads reached their maximum productivity fastest, after 3 days, but collapsed also, recovering after 4 days. The $\mathrm{CO}_{2}$ productivity was slightly delayed when casein was incorporated into $A \& K$ beads, but peaked at

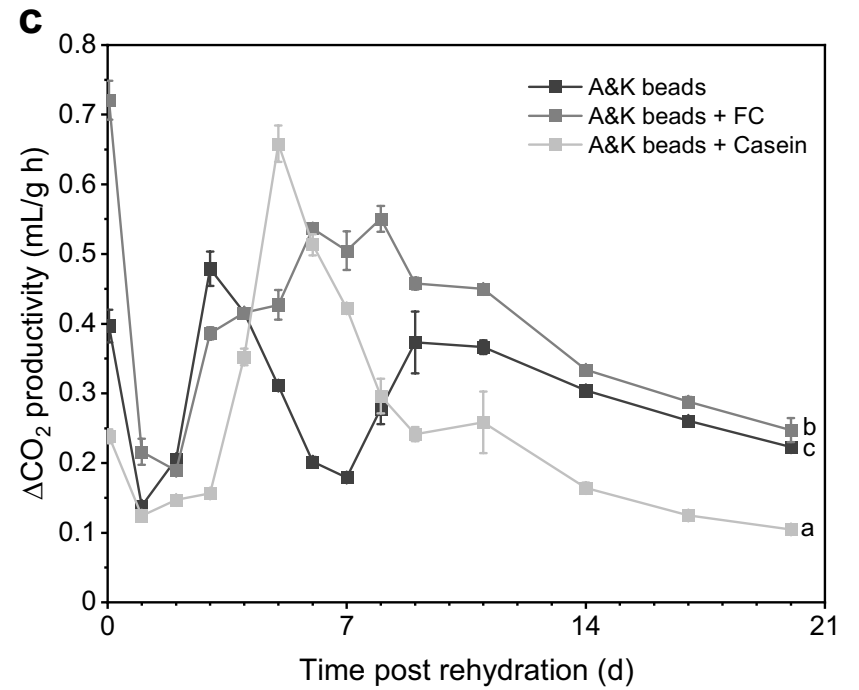

ity of M. brunneum and S. cerevisiae in Kill beads (b) or in A\&K beads (c) over time compared to beads without or with casein. Different letters indicate significant differences according to RM-ANOVA with Bonferroni post hoc test at $\mathrm{P}<0.05(\mathrm{n}=3)$

$0.65 \mathrm{~mL} / \mathrm{g}$ h after 5 days. Nevertheless, $\mathrm{CO}_{2}$ production also collapsed subsequently. In contrast, FC led to consistent production of $\mathrm{CO}_{2}$ across time, mitigating the negative effect, observed for plain A\&K beads. Therefore, A\&K beads with $\mathrm{FC}$ provided a higher $\mathrm{CO}_{2}$ release over a longer period of time. Moreover, $\mathrm{FC}$ enhanced the total $\mathrm{CO}_{2}$ produced by M. brunneum and S. cerevisiae in 20 days by $29 \%$ (from $142 \mathrm{~g} / \mathrm{L}$ to $183 \mathrm{~g} / \mathrm{L}$ ), whereas casein even decreased it by $19 \%$ (from $142 \mathrm{~g} / \mathrm{L}$ to $115 \mathrm{~g} / \mathrm{L}$ ). Thus, A\&K beads containing casein produced only $63 \%$ of the $\mathrm{CO}_{2}$ that $\mathrm{A} \& \mathrm{~K}$ beads containing FC released.

The replica confirmed these surprising results and FC increased the total $\mathrm{CO}_{2}$ yield even by $34 \%$ from $92 \mathrm{~mL} / \mathrm{g}$ to $123 \mathrm{~g} / \mathrm{L}$ (Online Resource 1, Fig. A6). FC increased the $\mathrm{CO}_{2}$ productivity of $M$. brunneum and probably $S$. cerevisiae over a period of 20 days. 
Even though incorporating FC did not reach the maximum $\mathrm{CO}_{2}$ productivity, it led to a more continuous production and hence to an increased total $\mathrm{CO}_{2}$ release.

We investigated whether the fluctuations in $\mathrm{CO}_{2}$ productivity of plain $A \& K$ beads were somehow caused by fluctuating water content or water activity. Beads were placed on semi selective water agar in Petri dishes and measured daily for 8 days. However, water absorption remained unchanged after $24 \mathrm{~h}$ for all formulations as they reached maximal water activity possible, which equaled that of moist beads (Online Resource 1, Fig. A7).

\section{Discussion}

In this study, we found that FC influences the porous properties of alginate/starch beads, thus enhancing their water absorbency. Moreover, FC increases the fungal activity of encapsulated $M$. brunneum and $S$. cerevisiae, leading to enhanced spore formation and $\mathrm{CO}_{2}$ productivity.

\section{$\mathrm{C} / \mathrm{N}$ ratio}

To assess the nutritional influence of FC, non-crosslinked casein was used as reference. Since both casein formulations differed only slightly in their carbon content, but noticeably in their nitrogen content, different impacts on sporulation must be taken into account. It is well known that type and concentration of carbon and nitrogen sources as well as the $\mathrm{C} / \mathrm{N}$ ratio impact growth and sporulation kinetics of fungi (Ajdari et al. 2011). In a study of Li and Holdom (1995), soluble starch supported growth and sporulation of Metarhizium anisopliae (M. anisopliae), and according to Braga et al. (1999) casein even reduced the lag phase and also increased the growth rate of $M$. anisopliae (Braga et al. 1999; Li and Holdom 1995). In the present study, incorporating casein led to a higher $\mathrm{C} / \mathrm{N}$ ratio compared to FC. M. anisopliae favored higher $\mathrm{C} / \mathrm{N}$ ratios of 160 , when sucrose as carbon and yeast extract as nitrogen source were used (Gao and Liu 2010), assuming that casein should offer more favorable nutritional conditions than FC.

\section{Rehydration vs. bead density, pore volume distribution and median pore size}

Rehydration kinetics were significantly influenced by casein and FC. The water absorbency was increased only by FC, reaching higher rehydration factors. However, the water activity was not enhanced and was even decreased by casein within the first hours. As the water activity reached its maximum, the water absorption still increased, thus, the porous structure of rehydrated beads was examined. Rehydrated beads with higher pore sizes should have shown a higher rehydration rate, which applies for FC but not for casein. The decreasing bead densities as well as the increasing median pore sizes and the tendency towards larger pore volumes are mutually supportive and strong indicators for the involvement of casein in the increased water absorption, although results were not as pronounced when casein was added. Rehydration involves different physical mechanisms such as capillary flow, convection and diffusion (Vreeker et al. 2008). According to Caccavo et al. (2018), the water transport in gel phases is complex and mainly driven by diffusion processes which however do not follow a pure Fickian behavior most of the time (Caccavo et al. 2018). FC, which is primarily investigated as tablet disintegrant, hardly swells during tablet disintegration. Non swelling agents are believed to impart their disintegration action through increased porosity and capillary action (Mohanachandran et al. 2011; Ponchel and Duchene 1990). Larger pore diameters result in faster capillary action with decreasing speed over time (Andersson et al. 2017). In this study, the latter is reflected by the rehydration factor which was increased by FC and decelerated over time. The mechanisms of capillary action in hydrogels are mainly based on water-water interaction. The reason why capillary flow even occurs is that water tends to lower its surface energy, by filling the pores with water so that the surface area exposed to air is reduced (Andersson et al. 2017). In contrast to FC, the water absorption of beads containing casein was decelerated compared to beads containing only starch, although the median pore size and bead density did not change. There is no explanation for this so far.

Overall, the pore size may be underestimated due to a considerable fraction of non-freezable water interacting with alginate/starch/casein, which is difficult to determine for hydrogels because the non-freezable water could exist not only on the pore surface but also in the matrix (Kazuhiko Ishikiriyama et al. 1995a, b). We assumed that this fraction is similar for each formulation, and may be assumed to equal $1.0 \mathrm{~nm}$ (Ishikiriyama et al. 1995a, b), and thus, determined pore sizes should be comparable. Moreover, the beads needed to be cut to fit into the aluminum pans, and to not exceed maximal weight. The smaller the sample size, and the bigger the contact area with the pan, the better the heat flux and the more accurate the measurement. Cutting surely disorganizes the porous structure, at least in the cutting zone, but we assumed that the potential error is smaller compared to that caused by a modified production process making microbeads. Results obtained from thermoporometry by DSC especially for polymers must be considered carefully and viewed with caution, because they can be affected by measurement conditions, which can cause e.g. water migration. Consequently, measurements made on heating can underestimate pore sizes whereas measurements made on cooling can overestimate them (Hay and Laity 
2000; Iza et al. 2000). However, pore size distributions of polymer membranes determined by thermoporometry were concluded to be valid and it was made clear that thermoporometry is a unique and the most reliable method to characterize mesoporous structures of hydrogels and pore sizes of polymer membranes, especially since it provides several advantages where classic methods such as mercury porometry fail or are limited to dry samples (Hay and Laity 2000; K. Ishikiriyama et al. 1995a, b; Iza et al. 2000; Riikonen et al. 2011). In the present study, the observation that results of replicates were quite consistent, strengthen the methods validity. The influence of the initial porosity of the dried system is of great interest, which however warrants a different methodology and may be subject of further investigations.

Rehydration experiments in this study were conducted under optimal conditions with maximum water availability, which does not reflect field conditions. Further studies under suboptimal conditions could reveal a stronger rehydration effect of FC. Moreover, rehydration depends on the ionic strength of the rehydration medium (Fang et al. 2011), thus, alginate barely swells in pure water. Its rehydration depends on the electrolyte solutions and increases with increasing salt concentrations, until disintegration occurs (Vreeker et al. 2008). Adding salts also influences the water binding by proteins because of their implications on electrostatic interactions (Zayas 1997). In this study, beads were rehydrated in ultra-pure water, so it can be assumed that water absorption is further favored in other media or by soil water during field application.

\section{Activity of Metarhizium brunneum and Saccharomyces cerevisiae}

FC enhanced the activity of $M$. brunneum more than casein, as revealed by increased sporulation. In view of our observation on pore structure and water absorption, we attribute the increased spore density to the altered network structure caused by FC. The effect was even more pronounced when the system was aerated, although beads were cultivated at a lower temperature $\left(23{ }^{\circ} \mathrm{C}\right.$ instead of $\left.25{ }^{\circ} \mathrm{C}\right)$ due to logistic reasons, and M. brunneum growth is known to be temperature dependent. This indicates that gas exchange like oxygen supply plays an important role which might be favored in the more porous structure conveyed by FC.

Interestingly, FC's effect on spore yield decreased once $S$. cerevisiae was co-encapsulated, whereas casein's impact was slightly improved on average, though spore yields were inconsistent. It is conceivable that $S$. cerevisiae compensated for the influence of $\mathrm{FC}$ on the porous structure due to its relatively high proportion in the matrix. We observed, that $S$. cerevisiae proliferation was favored on/in beads containing casein. However, stronger proliferation of $S$. cerevisiae coincided with poor sporulation of $M$. brunneum. There might have been an interaction between casein and $S$. cerevisiae, which led to inhibition of spore formation. It has already been reported that yeast, among them $S$. cerevisiae, are capable of decreasing the growth of filamentous spoilage fungi (Armando et al. 2012; Petersson and Schnürer 1995) and that actively growing yeast may affect the development of other microorganisms by acidifying the environment (Walker 1998), or by competing for space and nutrients (Armando et al. 2012). Moreover, active M. brunneum and S. cerevisiae may compete for oxygen as oxygen consumption rises. $M$. brunneum conidia production was increased by aeration, but only when FC was incorporated, which suggests that FC has a major influence on the alginate network and thus on oxygen diffusion, which impacts the proliferation of both fungi (Braga et al. 1999; Gosmann and Rehm 1986). Garcia-Oritz et al. (2015) found that the conidial production of $M$ anisopliae is enhanced and accelerated by oxygen-enriched pulses without impairing conidia quality (Garcia-Ortiz et al. 2015).

Moreover, it must be noted that the nutritional values of alginate/starch beads were measured only. In A\&K beads the impact of (dead) S. cerevisiae as nutrient supply must be considered, since yeast cells are rich nutrient sources, comprising 40-60\% of their biomass protein (Walker 1998). It is worth mentioning that nutritional conditions can alter the germination speed, adhesion and pathogen virulence of aerial conidia (Rangel et al. 2008), which should be considered in virulence tests and field trials.

Overall, we have not yet resolved, how the fungus develops inside the beads and how growth is influenced, since we investigated sporulation only.

It is known that co-encapsulating $S$. cerevisiae and the entomopathogenic fungus Beauveria bassiana enhances the $\mathrm{CO}_{2}$ release of moist alginate/starch beads compared to the respective single encapsulation (Vemmer et al. 2016), which is in line with our findings and emphasizes the importance of co-encapsulating S. cerevisiae. By adding $\mathrm{FC}, \mathrm{CO}_{2}$ was produced more constantly compared to regular $\mathrm{A} \& \mathrm{~K}$ beads, containing only starch. Why the $\mathrm{CO}_{2}$ productivity of these beads collapsed between 4 and 7 days remains unclear, but both caseins seemed to mitigate the negative effect. In the study of Vemmer et al. (2016) no decrease in $\mathrm{CO}_{2}$ productivity has been observed when $B$. bassiana was encapsulated, indicating a possible impact of M. brunneum. However, it must be noted that moist beads were investigated in their study. Despite, in our study, maximum $\mathrm{CO}_{2}$ was produced faster, which is in line with Humbert et al. (2017) who also studied, the $\mathrm{CO}_{2}$ release of dried alginate/starch beads, but depending on amyloglucosidase content. There, maximum $\mathrm{CO}_{2}$ concentration was reached after 7 days. This is in line with our results obtained from $\mathrm{A} \& \mathrm{~K}$ beads containing casein 
or FC. However, fluctuations in $\mathrm{CO}_{2}$ concentrations were not observed, even in the absence of amyloglucosidase (Humbert et al. 2017b).

Taking these studies together, there is nothing to indicate that drying and rehydration had any negative effect or, that amylase was lacking, when M. brunneum was encapsulated. It rather suggests that there is complex interaction between the amylase producing M. brunneum and the $\mathrm{CO}_{2}$ producing $S$. cerevisiae, which might also be nutrient mediated by casein. Furthermore, visible spore formation coincided with highest $\mathrm{CO}_{2}$ productivity of $\mathrm{A} \& \mathrm{~K}$ beads containing FC. The increased maximal $\mathrm{CO}_{2}$ release by FC coincided with enhanced conidia production, thus, better attraction of wireworms within the first week and enhanced probability of killing may possibly be achieved.

There are two major limitations of this study. Although non-crosslinked casein was used as reference, we cannot rule out an additional nutritive impact of FC, since casein and FC slightly differed in their nutritional content. However, adjusting the FC content to equal the nutritional content of casein, might also change the bead structure, thus, one cannot be considered separately from the other.

Secondly, we could not determine the porous properties of Kill beads or A\&K beads and thus, we can only assume, that the impact of FC on the bead structure is similar. Determining the pore volume distribution and pore sizes by thermoporometry requires salt-free samples, since the measuring principle is based on the melting point depression of pore water and can thus interfere with the measurement. More work has to be done to implement a valid method for alginate beads containing living cells. Nevertheless, the measurements regarding pore size and the impact of aeration on fungal activity are strong indicators for an altered bead structure that should be confirmed by other methods such as SEM.

In conclusion, FC influenced the porous properties of alginate/starch beads, possibly by implementing an altered network structure, thus enhancing their water absorbency. Moreover, FC increased the fungal activities of encapsulated M. brunneum and S. cerevisiae, resulting in enhanced $\mathrm{CO}_{2}$ productivity and increased spore yields.

Our data suggest that FC enhances the oxygen diffusion through modified pore structure, since aeration enhanced the fungal development. It is recommended to verify the altered oxygen diffusion using e.g. oxygen microelectrodes. Moreover, enlightening the complex symbiotic interaction of M. brunneum and S. cerevisiae is of great interest to further adjust the bead formulation. The obtained findings can be applied to other biological crop protection products, especially other microbial pest control agents.

Supplementary Information The online version contains supplementary material available at https://doi.org/10.1007/s11274-021-03121-3.
Acknowledgements We would like to thank Prof. Dr. Stefan Vidal (Agricultural Entomology, Department for Crop Science, GeorgAugust-University Goettingen, Germany) for providing M. brunneum strain CB15 and also T. Dellweg from Deutsche Hefewerke GmbH (Nürnberg, Germany) for providing S. cerevisiae strain H205. We are also grateful for the opportunity to use the DSC equipment from the WG of Prof. Dr.-Ing. Hüsgen (Plastics Engineering, Department of Engineering and Mathematics, Bielefeld University of applied sciences, Germany). Finally, we would like to thank Turid Frahnow (Data Science, Faculty of Economics, Bielefeld University, Germany) for answering any questions on statistics.

Author contributions All authors contributed to the study conception and design. Material preparation, data collection and analysis were performed by KMH. The first draft of the manuscript was written by $\mathrm{KMH}$ and all authors commented on previous versions of the manuscript. All authors read and approved the final manuscript.

Funding Open Access funding enabled and organized by Projekt DEAL. This study was funded by the German Federal Ministry of Food and Agriculture (BMEL) as part of the project ATTRACAP (No. 2814906815).

\section{Declarations}

Conflict of interest The authors have no conflicts of interest to declare that are relevant to the content of this article. The authors alone are responsible for the content and writing of the paper.

Open Access This article is licensed under a Creative Commons Attribution 4.0 International License, which permits use, sharing, adaptation, distribution and reproduction in any medium or format, as long as you give appropriate credit to the original author(s) and the source, provide a link to the Creative Commons licence, and indicate if changes were made. The images or other third party material in this article are included in the article's Creative Commons licence, unless indicated otherwise in a credit line to the material. If material is not included in the article's Creative Commons licence and your intended use is not permitted by statutory regulation or exceeds the permitted use, you will need to obtain permission directly from the copyright holder. To view a copy of this licence, visit http://creativecommons.org/licenses/by/4.0/.

\section{References}

Ajdari Z, Ebrahimpour A, Manan MA, Hamid M, Mohamad R, Ariff AB (2011) Nutritional requirements for the improvement of growth and sporulation of several strains of Monascus purpureus on solid state cultivation. J Biomed Biotechnol. https://doi.org/ $10.1155 / 2011 / 487329$

Andersson J, Ström A, Gebäck T, Larsson A (2017) Dynamics of capillary transport in semi-solid channels. Soft Matter 13:2562-2570. https://doi.org/10.1039/C6SM02305C

Arce CC, Theepan V, Schimmel BC, Jaffuel G, Erb M, Machado RA (2021) Plant-associated $\mathrm{CO}_{2}$ mediates long-distance host location and foraging behaviour of a root herbivore. eLife 10:e65575. https://doi.org/10.7554/eLife.65575

Armando MR, Dogi CA, Rosa CAR, Dalcero AM, Cavaglieri LR (2012) Saccharomyces cerevisiae strains and the reduction of Aspergillus parasiticus growth and aflatoxin $\mathrm{B}_{1}$ production at different interacting environmental conditions, in vitro. Food Addit 
Contam Part A 29:1443-1449. https://doi.org/10.1080/19440049. 2012.698655

Bajpai SK, Shah FF, Bajpai M (2017) Dynamic release of gentamicin sulfate (GS) from alginate dialdehyde (AD)-crosslinked casein (CAS) films for antimicrobial applications. Des Monomers Polym 20:18-32. https://doi.org/10.1080/15685551.2016.1231037

Braga GUL, Destéfano RHR, Messias CL (1999) Oxygen Consumption by Metarhizium anisopliae during Germination and Growth on Different Carbon Sources. J Invertebr Pathol 74:112-119. https:// doi.org/10.1006/jipa.1999.4872

Brandl MA, Schumann M, Przyklenk M, Patel A, Vidal S (2017) Wireworm damage reduction in potatoes with an attract-and-kill strategy using Metarhizium brunneum. J Pest Sci 90:479-493. https:// doi.org/10.1007/s10340-016-0824-x

Caccavo D, Cascone S, Lamberti G, Barba AA (2018) Hydrogels: experimental characterization and mathematical modelling of their mechanical and diffusive behaviour. Chem Soc Rev 47:2357-2373. https://doi.org/10.1039/C7CS00638A

Chan AW, Whitney RA, Neufeld RJ (2008) Kinetic controlled synthesis of $\mathrm{pH}$-responsive network alginate. Biomacromolecules 9:2536-2545. https://doi.org/10.1021/bm800594f

Elzoghby AO, Abo El-Fotoh WS, Elgindy NA (2011) Casein-based formulations as promising controlled release drug delivery systems. J Control Release 153:206-216. https://doi.org/10.1016/j. jconrel.2011.02.010

European Food Safety Authority (2013) Conclusion on the peer review of the pesticide risk assessment for bees for the active substance fipronil. EFSA J 11:3158. https://doi.org/10.2903/j.efsa.2013.3158

Fang Y, Li L, Vreeker R, Yao X, Wang J, Ma Q, Jiang F, Phillips GO (2011) Rehydration of dried alginate gel beads: effect of the presence of Gelatin and Gum Arabic. Carbohydr Polym 86:11451150. https://doi.org/10.1016/j.carbpol.2011.06.003

Fekete, Suemegh, Hoffmann, Janos, Denes, 1990. Verfahren zur Herstellung von Mikrokapseln, welche eine schnelle Abgabe des Wirkstoffes aus ihnen beziehungsweise den aus ihnen herstellbaren Tabletten und die unmittelbare Tablettierung von Arzneimittelwirkstoffen gewaehrleisten. DE3943338.

Gao L, Liu X (2010) Nutritional requirements of mycelial growth and sporulation of several biocontrol fungi in submerged and on solid. Culture 79:8. https://doi.org/10.1134/S002626171005005X

Garcia-Ortiz N, Tlecuitl-Beristain S, Favela-Torres E, Loera O (2015) Production and quality of conidia by Metarhizium anisopliae var. lepidiotum: critical oxygen level and period of mycelium competence. Appl Microbiol Biotechnol 99:2783-2791. https://doi.org/ 10.1007/s00253-014-6225-2

Gosmann B, Rehm H (1986) Oxygen uptake of microorganisms entrapped in Ca-alginate. Appl Microbiol Biotechnol. https://doi. org/10.1007/BF00261907

Hay JN, Laity PR (2000) Observations of water migration during thermoporometry studies of cellulose films. Polymer 41:6171-6180. https://doi.org/10.1016/S0032-3861(99)00828-9

He Z, Zhang X, Qi W, Huang R, Su R (2015) Alginate-casein microspheres as bioactive vehicles for nutrients. Trans Tianjin Univ 21:383-391. https://doi.org/10.1007/s12209-015-2692-5

Humbert P, Przyklenk M, Vemmer M, Schumann M, Vidal S, Patel AV (2017a) Technical scale production of encapsulated Saccharomyces cerevisiae and Metarhizium brunneum attractive to wireworms. Biocontrol Sci Tech 27:1049-1070. https://doi.org/ 10.1080/09583157.2017.1380164

Humbert P, Vemmer M, Giampà M, Bednarz H, Niehaus K, Patel AV (2017b) Co-encapsulation of amyloglucosidase with starch and Saccharomyces cerevisiae as basis for a long-lasting $\mathrm{CO} 2$ release. World J Microbiol Biotechnol 33:71. https://doi.org/10. 1007/s11274-017-2237-2

Ishikiriyama K, Sakamoto A, Todoki M, Tayama T, Tanaka K, Kobayashi T (1995a) Pore size distribution measurements of polymer hydrogel membranes for artificial kidneys using differential scanning calorimetry. Thermochim Acta 267:169-180. https://doi.org/ 10.1016/0040-6031(95)02476-X

Ishikiriyama K, Todoki M, Motomura K (1995b) Pore size distribution (PSD) measurements of silica gels by means of differential scanning calorimetry: I. Optimization for determination of PSD. J Colloid Interface Sci 171:92-102. https://doi.org/10.1006/jcis. 1995.1154

Iza M, Woerly S, Danumah C, Kaliaguine S, Bousmina M (2000) Determination of pore size distribution for mesoporous materials and polymeric gels by means of DSC measurements: thermoporometry. Polymer 41:5885-5893. https://doi.org/10.1016/S00323861(99)00776-4

Kim Y-J, Yoon K-J, Ko S-W (2000) Preparation and properties of alginate superabsorbent filament fibers crosslinked with glutaraldehyde. J Appl Polym Sci 78:1797-1804. https://doi.org/10. 1002/1097-4628(20001205)78:10\%3c1797::AID-APP110\%3e3.0. $\mathrm{CO} ; 2-\mathrm{M}$

Krell V, Jakobs-Schoenwandt D, Vidal S, Patel AV (2018) Encapsulation of Metarhizium brunneum enhances endophytism in tomato plants. Biol Control 116:62-73. https://doi.org/10.1016/j.bioco ntrol.2017.05.004

Kulkarni AR, Soppimath KS, Aminabhavi TM, Dave AM, Mehta MH (2000) Glutaraldehyde crosslinked sodium alginate beads containing liquid pesticide for soil application. J Control Release 63:97-105. https://doi.org/10.1016/S0168-3659(99)00176-5

Li DP, Holdom DG (1995) Effects of nutrients on colony formation, growth, and sporulation of Metarhizium anisopliae (Deuteromycotina: Hyphomycetes). J Invertebr Pathol 65:253-260. https:// doi.org/10.1006/jipa.1995.1039

Maloney TC (2000) Messen der Porengrössenverteilung mittels DSC. UserCom Mettler Toledo 15-17

Mohanachandran PS, Sindhumol PG, Kiran TS (2011) Superdisintegrants: an overview. Int J Pharm Sci Rev Res 6:105-109

Mørch ÝA, Donati I, Strand BL, Skjåk-Bræk G (2007) Molecular engineering as an approach to design new functional properties of alginate. Biomacromolecules 8:2809-2814. https://doi.org/10. 1021/bm700502b

National research council of the United States of America (1930) International critical tables of Numerical Data, Physics, Chemistry and Technology. McGraw-Hill Book Company. https://doi.org/ $10.17226 / 20230$

Parker WE, Howard JJ (2001) The biology and management of wireworms (Agriotes spp.) on potato with particular reference to the U.K. Agric For Entomol 3:85-98. https://doi.org/10.1046/j.14619563.2001.00094.x

Patwa R, Zandraa O, Capáková Z, Saha N, Sáha P (2020) Effect of iron-oxide nanoparticles impregnated bacterial cellulose on overall properties of alginate/casein hydrogels: potential injectable biomaterial for wound healing applications. Polymers 12:2690. https://doi.org/10.3390/polym12112690

Petersson S, Schnürer J (1995) Biocontrol of mold growth in highmoisture wheat stored under airtight conditions by Pichia anomala, Pichia guilliermondii, and Saccharomyces cerevisiae. Appl Environ Microbiol 61:1027-1032

Poggi S, Le Cointe R, Riou J-B, Larroudé P, Thibord J-B, Plantegenest M (2018) Relative influence of climate and agroenvironmental factors on wireworm damage risk in maize crops. J Pest Sci 91:585-599. https://doi.org/10.1007/s10340-018-0951-7

Ponchel G, Duchene D (1990) Evaluation of formalin-casein as a tablet disintegrant. Drug Dev Ind Pharm 16:613-628. https://doi.org/10. 3109/03639049009104406

Przyklenk M, Vemmer M, Hanitzsch M, Patel A (2017) A bioencapsulation and drying method increases shelf life and efficacy of Metarhizium brunneum conidia. J Microencapsul 34:498-512. https://doi.org/10.1080/02652048.2017.1354941 
Riikonen J, Salonen J, Lehto V-P (2011) Utilising thermoporometry to obtain new insights into nanostructured materials: Review part 2. J Therm Anal Calorim 105:823-830. https://doi.org/10.1007/ s10973-011-1337-8

Schindelin J, Arganda-Carreras I, Frise E, Kaynig V, Longair M, Pietzsch T, Preibisch S, Rueden C, Saalfeld S, Schmid B, Tinevez J-Y, White DJ, Hartenstein V, Eliceiri K, Tomancak P, Cardona A (2012) Fiji: an open-source platform for biological-image analysis. Nat Methods 9:676-682. https://doi.org/10.1038/nmeth.2019

Schumann M, Patel A, Vidal S (2014) Soil application of an encapsulated $\mathrm{CO} 2$ source and its potential for management of Western Corn Rootworm Larvae. J Econ Entom 107:230-239. https://doi. org/10.1603/EC13344

Vemmer M, Schumann M, Beitzen-Heineke W, French BW, Vidal S, Patel AV (2016) Development of a CO2-releasing coformulation based on starch, Saccharomyces cerevisiae and Beauveria bassiana attractive towards western corn rootworm larvae. Pest Manag Sci 72:2136-2145. https://doi.org/10.1002/ps.4245
Vernon RS, van Herk WG (2013) Wireworms as pests of potato. Insect Pests of Potato. https://doi.org/10.1016/B978-0-12-386895-4. 00005-3

Vreeker R, Li L, Fang Y, Appelqvist I, Mendes E (2008) Drying and rehydration of calcium alginate gels. Food Biophys 3:361-369. https://doi.org/10.1007/s11483-008-9087-2

Walker GM (1998) Yeast physiology and biotechnology. John Wiley \& Sons

Zayas JF (1997) Water holding capacity of proteins. In: Zayas JF (ed) Functionality of proteins in food. Springer, Berlin, pp 76-133. https://doi.org/10.1007/978-3-642-59116-7_3

Publisher's Note Springer Nature remains neutral with regard to jurisdictional claims in published maps and institutional affiliations.

\section{Authors and Affiliations}

\section{Katharina M. Hermann ${ }^{1,2} \cdot$ Alexander Grünberger $^{2} \cdot$ Anant V. Patel $^{1}$}

1 Faculty of Engineering and Mathematics, Fermentation and Formulation of Biologicals and Chemicals, Bielefeld University of Applied Sciences, Bielefeld, Germany
2 Faculty of Technology, Multiscale Bioengineering, Bielefeld University, Bielefeld, Germany 